\title{
On Some Multidimensional Limit Boundary Value Problems
}

\author{
V. B. Vasilyev ${ }^{1 *}$ and Sh. H. Kutaiba ${ }^{1 * *}$ \\ (Submitted by A. B. Muravnik) \\ ${ }^{1}$ Chair of Applied Mathematics and Computer Modeling, Belgorod State National Research University, \\ Belgorod, 308015 Russia \\ Received December 24, 2020; revised February 18, 2021; accepted February 24, 2021
}

\begin{abstract}
We study solvability for a model elliptic pseudo-differential equation with an additional integral condition in Sobolev-Slobodetskii spaces in certain conical domains in Euclidean space. . Using the wave factorization for an elliptic symbol we construct a solution for this boundary value problem and study the behavior of the solution when parameters of cones tend to their extremal values. It was shown that for such a solvability we need certain additional condition besides presented integral condition.
\end{abstract}

DOI: $10.1134 / \mathrm{S} 1995080221060317$

Keywords and phrases: elliptic pseudo-differential equation, boundary value problem, singular domain, asymptotic behavior.

\section{INTRODUCTION}

This paper is related to special boundary value problems for pseudo-differential equation in nonordinary singular domains of Euclidean space. Such domains we treat as cones in a space of lower dimension and we construct these domains by limit state of bigger cones.

Contrasting to other approaches to boundary value problems for (pseudo)differential equations (see, for example, [1,2] and related books) we use other concepts which were introduced in author's book [9] (see also [14]). Our approach is closely related to the theory of boundary value problems for elliptic pseudo-differential equations described in [3].

We describe here basic ideas using very simple example pictured in Fig. 1. We can find a general solution for a model pseudo-differential equation in such a domain and also to extract the unique solution for certain additional conditions, We are interested in a behavior of the solution under $\alpha \rightarrow 0$, i.e. $a \rightarrow \infty$; in this case we obtain the domain pictured in Fig. 2. Some results were obtained by the authors in this direction. But there are multidimensional more complicated singularities (see below) for which the same questions are valid. We consider here some combination of such singularities and establish certain conditions for solvability of considered problems.

\section{EQUATIONS AND SOLVABILITY}

We use a local principle which asserts that for Fredholm property of a general pseudo-differential equation with the symbol $A(x, \xi)$ we need to obtain invertibility property for a model pseudo-differential equation with the symbol $A(\cdot, \xi)$.

\footnotetext{
${ }^{*}$ E-mail: vbv57@inbox.ru

${ }^{* *}$ E-mail: $1167542 @ b s u . e d u . r u$
} 


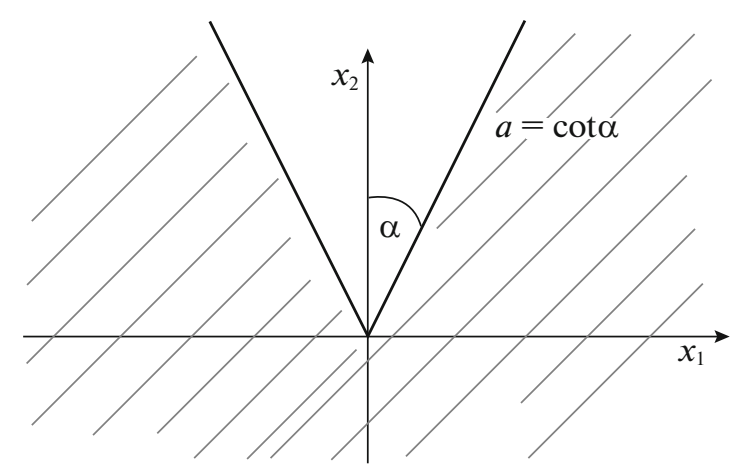

Fig. 1. An exterior angle.

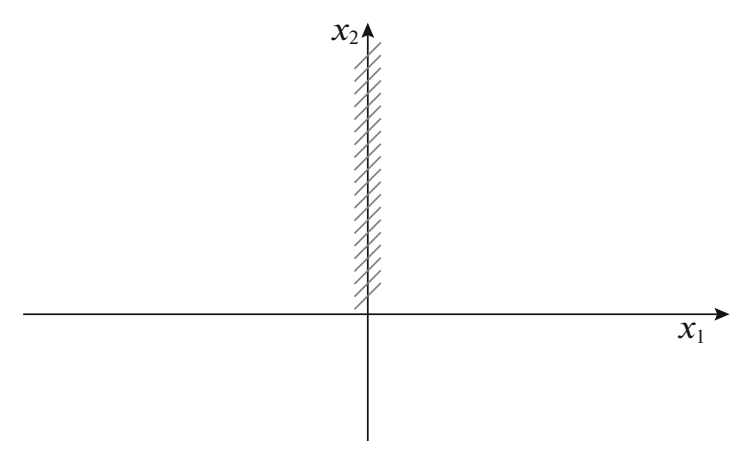

Fig. 2. A half-infinity crack in a plane.

\subsection{Model Equations and Canonical Domains}

Definition 1. A domain $D \subset \mathbb{R}^{m}$ is called a canonical domain if it is certain simply connected cone.

Example 1. Two standard domains $\mathbb{R}^{m}$ and half-space $\mathbb{R}_{+}^{m}=\left\{x \in \mathbb{R}^{m}: x=\left(x^{\prime}, x_{m}\right), x_{m}>0\right\}$ are typical simply connected cones.

Let $A(x, \xi)$ de a function defined on $\mathbb{R}^{m} \times \mathbb{R}^{m}$ such that the integral

$$
\int_{\mathbb{R}^{m}} \int_{\mathbb{R}^{m}} A(x, \xi) e^{i(x-\xi)} u(y) d y d \xi
$$

exists at least for $u \in S\left(\mathbb{R}^{m}\right)$, where $S\left(\mathbb{R}^{m}\right)$ is the Schwartz class [3].

Definition 2. The operator

$$
u(x) \longmapsto \int_{\mathbb{R}^{m}} \int_{\mathbb{R}^{m}} A(x, \xi) e^{i(x-\xi)} u(y) d y d \xi
$$

is called a pseudo-differential operator $A$ with the symbol $A(x, \xi)$.

Definition 3. A model pseudo-differential equation in a canonical domain $D$ is called the following equation

$$
(A u)(x)=v(x), \quad x \in D,
$$

where a symbol of the operator $A$ doesn't depend on $x, A(x, \xi) \equiv A(\xi)$, and unknown function $u$ is defined in the domain $D$ only.

We will consider below symbols satisfying the condition

$$
c_{1}(1+|\xi|)^{\alpha} \leq|A(\xi)| \leq c_{2}(1+|\xi|)^{\alpha}, \quad \alpha \in \mathbb{R},
$$

with positive constants $c_{1}, c_{2}$. 
The equation (1) is studied in the space $H^{s}(D)$; it is subspace of $H^{s}\left(\mathbb{R}^{m}\right)$ with respect to the norm

$$
\|u\|_{s}=\left(\int_{\mathbb{R}^{m}}|\tilde{u}(\xi)|^{2}(1+|\xi|)^{2 s} d \xi\right)^{1 / 2},
$$

where $\tilde{u}$ is the Fourier transform of the function $u$,

$$
\tilde{u}(\xi)=\int_{\mathbb{R}^{m}} e^{i x \cdot \xi} u(x) d x,
$$

and vanishing right hand side $v \equiv 0$.

\subsection{Basic Constructions}

We will describe here a general scheme for obtaining different singular domains in multidimensional Euclidean space. These singular domain are complications of domains considered earlier by the author.

Let $D \equiv C_{+}$be a convex cone in $\mathbb{R}^{m}$ non-including a whole straight line, $C_{-} \equiv-C_{+}$. To describe a solution of a homogeneous equation (1) we use a special factorization for an elliptic symbol, so called wave factorization [9]. It is a representation of the $\operatorname{symbol} A(\xi)$ as a product $A(\xi)=A_{\neq}(\xi)$. $A_{=}(\xi)$, where the factors admit holomorphic continuation into radial tube domains $T\left({\stackrel{*}{C_{+}}}^{*}, T\left(\mathcal{C}_{-}^{*}\right)\right.$ respectively [6], $\stackrel{*}{C}$ denotes conjugate cone with respect to the cone $C$,

$$
\stackrel{*}{C}=\left\{x \in \mathbb{R}^{m}: x \cdot y>0, \forall y \in C\right\} .
$$

Remark 1. Such a factorization is applicable when we consider the equation (1) in the domain $\mathbb{R}^{m} \backslash C_{+}$.

Lemma 1. Let $C_{1} \subset \mathbb{R}^{m}, C_{2} \subset \mathbb{R}^{l}$ be convex cones non-including whole straight lines in $\mathbb{R}^{m}, \mathbb{R}^{l}$ respectively. Then $C=C_{1} \times C_{2}$ is a convex cone non-including a whole line in $\mathbb{R}^{m+l}$.

Proof. By contradiction. Indeed, if we assume that we have a straight line $\ell \subset C_{1} \times C_{2}$ and it goes across the point $\left(x_{0}, y_{0}\right) \in C$ then all points $\lambda x_{0}, \forall \lambda \in \mathbb{R}$ should belong to the cone $C_{1}$; it is impossible by our assumption. A convexity is verified immediately.

Let us remind that here an important role plays the theory of one-dimensional singular integral operators $[4,5,7,8]$.

Below we will consider the following conical domain $D=\mathbb{R}^{m+n} \backslash\left(C_{1} \times C_{2}\right)$ and use the coordinates $x=\left(x_{1}, \cdots, x_{m}\right) \in \mathbb{R}^{m}, y=\left(y_{1}, \cdots, y_{n}\right) \in \mathbb{R}^{n}$ and dual coordinates for Fourier images $\xi=$ $\left(\xi_{1}, \cdots, \xi_{m}\right) \in \mathbb{R}^{m}$ and $\eta=\left(\eta_{1}, \cdots, \eta_{n}\left(\in \mathbb{R}^{n}\right.\right.$ respectively.

We will assume below that the surface $\partial C_{1}$ is given by the equation $x_{m}=\phi\left(x^{\prime}\right)$ and the surface $\partial C_{2}$ by the equation $y_{n}=\psi\left(y^{\prime}\right), x^{\prime}=\left(x_{1}, \ldots, x_{m-1}\right), y^{\prime}=\left(y_{1}, \ldots, y_{n-1}\right)$ so that the cone $C$ under consideration has coordinates $(x, y)$. We assume also the functions $\phi, \psi$ are continuously differentiable everywhere excluding the origin and $\phi(0)=\psi(0)=0$. Let us introduce the change of variables

$$
\left\{\begin{array}{l}
t_{1}=x_{1} \\
t_{2}=x_{2} \\
\cdots \\
t_{m-1}=x_{m-1} \\
t_{m}=x_{m}-\phi\left(x^{\prime}\right)
\end{array}, \quad\left\{\begin{array}{l}
\tau_{1}=y_{1} \\
\tau_{2}=y_{2} \\
\cdots \\
\tau_{n-1}=y_{n-1} \\
\tau_{n}=y_{n}-\psi\left(y^{\prime}\right)
\end{array}\right.\right.
$$

and we denote this operator by $T_{\phi}: \mathbb{R}^{m} \rightarrow \mathbb{R}^{m}$ so that this operator preserves point of the lower halfspace and $\partial C_{1}$ transforms into hyperplane $t_{m}=0$. Analogously we define the operator $T_{\psi}: \mathbb{R}^{n} \rightarrow \mathbb{R}^{n}$ and denote $T_{\phi \psi}: \mathbb{R}^{m+n} \rightarrow \mathbb{R}^{m+n}$ which transforms $\partial C$ into hyperplane $x_{m}=y_{n}=0$. Obviously, $T_{\phi \psi}=T_{\phi} \cdot T_{\psi}=T_{\psi} \cdot T_{\phi}=T_{\psi \phi}$ because these operators act on distinct variables. The Jacobian $D(x, y) / D(t, \tau)=1$ almost everywhere, i.e. for all points in $\mathbb{R}^{m+n}$ excluding the hyperplanes $x_{m}=0$, 
$y_{n}=0$. Further, we denote $F_{1}$ the partial Fourier transform on first $m$ variables in $\mathbb{R}^{m+n}$, and $F_{2}$ on left $n$ variables so that the standard Fourier transform $F$ in $\mathbb{R}^{m+n}$ is the following: $F=F_{1} \cdot F_{2}=F_{2} \cdot F_{1}$.

For operators $T_{\phi}, T_{\psi}$ there are the transmutation operators [10-12]

$$
V_{\phi}=F_{1} T_{\phi} F_{1}^{-1}, \quad V_{\psi}=F_{2} T_{\phi} F_{2}^{-1}
$$

acting on first $m \mathrm{co}=$ variables and last $n$ co-variables respectively. Let us denote $V_{\phi \psi}=F T_{\phi \psi} F^{-1}$. It is easy to see that

$$
V_{\phi \psi}^{-1}=V_{\phi}^{-1} \cdot V_{\psi}^{-1}=V_{\psi}^{-1} \cdot V_{\phi}^{-1} .
$$

Using these observations we can obtain the following assertion.

Theorem 1. If the symbol $A(\xi, \eta)$ admits the wave factorization with respect to the cone $C=C_{1} \times C_{2}$ with the index $æ, \rightsquigarrow-s=n+\epsilon, n \in \mathbb{N},|\epsilon|<1 / 2$, then a general solution of the homogeneous equation (1) in Fourier images is given by the formula

$$
\tilde{u}_{+}(\xi)=+A_{\neq}^{-1}(\xi, \eta) V_{\phi \psi}^{-1}\left(\sum_{|k|=0}^{n-1} \tilde{c}_{k_{1} k_{2}}\left(\xi^{\prime}, \eta^{\prime}\right) \xi_{m}^{k_{1}} \eta_{n}^{k_{2}}\right),
$$

where $c_{k_{1} k_{2}}\left(x^{\prime}\right) \in H^{s_{k_{1} k_{2}}}\left(\mathbb{R}^{m+n-2}\right)$ are arbitrary functions, $s_{k_{1} k_{2}}=s-\Re+|k|+1,|k|=0,1, \ldots, n-$ $1,|k|=k_{1}+k_{2}$.

Proof. We use notations from our previous papers $[10,11]$ and existence of the wave factorization with respect to the bigger cone $C: A(\xi, \eta)=A_{\neq}(\xi, \eta) \cdot A_{=}(\xi, \eta)$ with the index $æ$. Since the operator $A$ acts as an linear bounded operator $H^{s}\left(\mathbb{R}^{m+n}\right) \rightarrow H^{s-\alpha}\left(\mathbb{R}^{m+n}\right)$ we denote

$$
u_{-}(x)=(A u)(x), \quad x \in \mathbb{R}^{m+n} \backslash \bar{C},
$$

assuming that $u_{-}(x)=0$ for $x \in C$. Then, we rewrite the equation

$$
(A u)(x)-u_{-}(x)=0, \quad x \in \mathbb{R}^{m+n} .
$$

Applying the Fourier transform and the wave factorization we have

$$
A_{\neq}(\xi, \eta) \tilde{u}(\xi, \eta)=A_{=}^{-1}(\xi, \eta) \tilde{u}_{-}(\xi, \eta) .
$$

Now we have the following inclusions [9]

$$
A_{\neq}(\xi, \eta) \tilde{u}(\xi, \eta) \in \widetilde{H}^{s-æ}(C), \quad A_{=}^{-1}(\xi, \eta) \tilde{u}_{-}(\xi, \eta) \in \widetilde{H}^{s-æ}\left(\mathbb{R}^{m+n} \backslash \bar{C}\right) .
$$

Thus, we have

$$
F^{-1} A_{\neq}(\xi, \eta) \tilde{u}(\xi, \eta) \in H^{s-æ}(C), \quad F^{-1} A_{=}^{-1}(\xi, \eta) \tilde{u}_{-}(\xi, \eta) \in H^{s-æ}\left(\mathbb{R}^{m+n} \backslash \bar{C}\right),
$$

so that it is a unique distribution $f(x)$ supported on $\partial C$. Therefore, we obtain the equality

$$
F_{(\xi, \eta) \rightarrow(x, y)}^{-1}\left(A_{\neq}(\xi, \eta) \tilde{u}(\xi, \eta)\right)=f(x, y) .
$$

Acting by the operator $T_{\phi \psi}$ on left and right sides of the latter equality we obtain the distribution $f\left(T_{\phi} x, T_{\psi} y\right)$ supported on the hyperplane $t_{m}=\tau_{m}=0$.

According to the theory of distributions [6] we can conclude that

$$
f\left(T_{\phi} x, T_{\psi} y\right)=\sum_{|k|=0}^{l} c_{k_{1} k_{2}}\left(t^{\prime}, \tau^{\prime}\right) \delta^{k_{1}}\left(t_{m}\right) \otimes \delta^{k_{2}}\left(\tau_{n}\right),
$$

where $|k|=k_{1}+k_{2}, \delta$ is the Dirac mass-function.

Further, we now apply the Fourier transform in $\mathbb{R}^{m+n}$ and finally obtain

$$
F T_{\phi \psi} F^{-1}\left(A_{\neq}(\xi, \eta) \tilde{u}(\xi, \eta)\right)=\sum_{|k|=0}^{l} \tilde{c}_{k_{1} k_{2}}\left(\xi^{\prime}, \eta^{\prime}\right) \xi_{m}^{k_{1}} \eta_{n}^{k_{2}} .
$$


Taking into account the definition for $V_{\phi \psi}$ we can write

$$
\tilde{u}(\xi, \eta)=A_{\neq}(\xi, \eta) V_{\phi \psi}^{-1}\left(\sum_{|k|=0}^{l} \tilde{c}_{k_{1} k_{2}}\left(\xi^{\prime}, \eta^{\prime}\right) \xi_{m}^{k_{1}} \eta_{n}^{k_{2}}\right) .
$$

The left part of the proof is devoted to refine the value $l$ and $H^{s}$-spaces which include the functions $c_{k_{1} k_{2}}$. We starting from the fact that all summands $c_{k_{1} k_{2}}\left(\xi^{\prime}, \eta^{\prime}\right) \xi_{m}^{k_{1}} \eta_{n}^{k_{2}}$ should belong to the space $H^{s-æ}\left(\mathbb{R}^{m+n}\right)$. If so we can estimate

$$
\begin{aligned}
& \int_{\mathbb{R}^{m+n}}\left|c_{k_{1} k_{2}}\left(\xi^{\prime}, \eta^{\prime}\right) \xi_{m}^{k_{1}} \eta_{n}^{k_{2}}\right|^{2}(1+|\xi|+|\eta|)^{2(s-æ)} d \xi d \eta \\
\leq & c \int_{\mathbb{R}^{m+n}}\left|c_{k_{1} k_{2}}\left(\xi^{\prime}, \eta^{\prime}\right)\right|^{2}(1+|\xi|+|\eta|)^{2(s-æ+|k|)} d \xi d \eta,
\end{aligned}
$$

and for existence the double integral

$$
\int_{\mathbb{R}^{2}}(1+|\xi|+|\eta|)^{2(s-æ+|k|)} d \xi_{m} d \eta_{n}
$$

a necessary condition is the following: $2(s-æ+|k|)<-2$. We remind here that $æ-s=n+\epsilon,|\epsilon|<$ $1 / 2$, so that $|k|<n+\epsilon-1$, and we conclude that maximal value for $|k|$ is $n-1$; it is possible for $\epsilon>0$.

From above estimates we find that $c_{k_{1} k_{2}} \in H^{s_{k_{1}} k_{2}}\left(\mathbb{R}^{m+n-2}\right)$, where $s_{k_{1} k_{2}}=s-\mathfrak{x}+|k|+1,|k|=$ $0,1, \cdots, n-1$.

Remark 2. In this proof we take into account that $A_{\neq}$corresponds to $-C$ and $A_{=}$corresponds to $C$ (see details in [9]).

Obviously, the assertion of Theorem 1 is valid for the cone $C=C_{1} \times C_{2} \times \cdots \times C_{k}$ in the space $\mathbb{R}^{d}$, where $C_{l} \subset \mathbb{R}^{n_{l}}, d=\sum_{l=1}^{k} n_{l}$.

Of course, Theorem 1 is a very general, but we will consider below certain concrete cones for which we can present exact evaluations of operators $V_{\phi \psi}$. For certain convex cones such calculations are given in [10-13] and we will use these results for describing new singularities.

\subsubsection{Certain canonical domains}

a. m-dimensional circle cone. This cone has the form

$$
C_{+}^{a}=\left\{x \in \mathbb{R}^{m}: x=\left(x^{\prime}, x_{m}\right), x_{m}>a\left|x^{\prime}\right|, a>0\right\} .
$$

b. 4-faced angle in 3-dimensional space. This cone has the form

$$
C_{+}^{a b}=\left\{x \in \mathbb{R}^{3}: x=\left(x_{1}, x_{2}, x_{4}\right), x_{3}>a\left|x_{1}\right|+\left|x_{2}\right|, a, b>0\right\} .
$$

c. $m$-faced angle in $m$-dimensional space. This cone has the form

$$
C_{+}=\left\{x \in \mathbb{R}^{m}: x_{k}>0, k=1, \ldots, m\right\} .
$$

Let us note for the cone we have no parameters.

2.2.2. Description of operators $\boldsymbol{V}_{\boldsymbol{\phi}}, \boldsymbol{V}_{\boldsymbol{\psi}}$. The cases a) and b) from 2.2.1 will help us to construct new canonical singularities of lower dimensions when some parameters of cones tend to infinity. For example, Fig. 3 corresponds to the case b) when $a$ or $b$ tends to $\infty$ so we obtain 3 -dimensional domain with a cut as a 2 -dimensional half-plane.

The first operator $V_{\phi}$ is related to 2 -dimensional cone.

The case $m=2$ is a very good, there is only one mentioned cone. We write it as follows

$$
C_{+}^{a}=\left\{x \in \mathbb{R}^{2}: x=\left(x_{1}, x_{2}\right), x_{2}>a\left|x_{1}\right|, a>0\right\},
$$




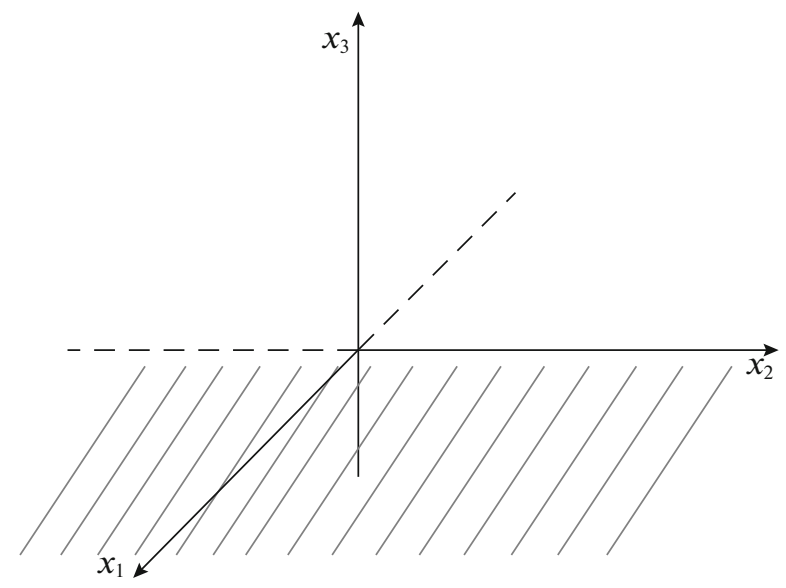

Fig. 3. A half-plane cut in a space.

and further evaluate:

$$
\begin{gathered}
\left(F_{1} T_{\varphi}^{-1} u\right)(\xi)=\int_{-\infty}^{+\infty} e^{i a\left|y_{1}\right| \xi_{2}} e^{i y_{1} \xi_{1}} \hat{u}\left(y_{1}, \xi_{2}\right) d y_{1} \\
=\int_{-\infty}^{+\infty} \chi_{+}\left(y_{1}\right) e^{i a y_{1} \xi_{2}} e^{i y_{1} \xi_{1}} \hat{u}\left(y_{1}, \xi_{2}\right) d y_{1}+\int_{-\infty}^{+\infty} \chi_{-}\left(y_{1}\right) e^{-i a y_{1} \xi_{2}} e^{i y_{1} \xi_{1}} \hat{u}\left(y_{1}, \xi_{2}\right) d y_{1} \\
=\int_{-\infty}^{+\infty} \chi_{+}\left(y_{1}\right) e^{i y_{1}\left(a \xi_{2}+\xi_{1}\right)} \hat{u}\left(y_{1}, \xi_{2}\right) d y_{1}+\int_{-\infty}^{+\infty} \chi_{-}\left(y_{1}\right) e^{-i y_{1}\left(a \xi_{2}-\xi_{1}\right)} \hat{u}\left(y_{1}, \xi_{2}\right) d y_{1}
\end{gathered}
$$

where $\hat{u}\left(y_{1}, \xi_{2}\right)$ denotes the one-dimensional Fourier transform with respect to a second variable, $\chi_{ \pm}$ are indicators of $\mathbb{R}_{ \pm}$. The latter two summands are Fourier transforms of functions $\chi_{+}\left(y_{1}\right) \hat{u}\left(y_{1}, \xi_{2}\right)$, $\chi_{-}\left(y_{1}\right) \hat{u}\left(y_{1}, \xi_{2}\right)$ with respect to a variable $y_{1}$ so that we we can use the following properties [3]; these properties are called Sokhotskii formulas [7,8]:

$$
\begin{aligned}
& \int_{-\infty}^{+\infty} \chi_{+}(x) e^{i x \xi} u(x) d x=\frac{1}{2} \tilde{u}(\xi)+v \cdot p \cdot \frac{i}{2 \pi} \int_{-\infty}^{+\infty} \frac{\tilde{u}(\eta) d \eta}{\xi-\eta} \\
& \int_{-\infty}^{+\infty} \chi_{-}(x) e^{i x \xi} u(x) d x=\frac{1}{2} \tilde{u}(\xi)-v \cdot p \cdot \frac{i}{2 \pi} \int_{-\infty}^{+\infty} \frac{\tilde{u}(\eta) d \eta}{\xi-\eta} .
\end{aligned}
$$

Taking into account these properties we conclude

$$
\begin{gathered}
\left(F_{1} T_{\varphi}^{-1} u\right)(\xi)=\frac{\tilde{u}\left(\xi_{1}+a \xi_{2}, \xi_{2}\right)+\tilde{u}\left(\xi_{1}-a \xi_{2}, \xi_{2}\right)}{2} \\
+v \cdot p \cdot \frac{i}{2 \pi} \int_{-\infty}^{+\infty} \frac{\tilde{u}\left(\eta, \xi_{2}\right) d \eta}{\xi_{1}+a \xi_{2}-\eta}-v \cdot p \cdot \frac{i}{2 \pi} \int_{-\infty}^{+\infty} \frac{\tilde{u}\left(\eta, \xi_{2}\right) d \eta}{\xi_{1}-a \xi_{2}-\eta} \equiv\left(V_{\varphi}^{-1} \tilde{u}\right)(\xi) .
\end{gathered}
$$

Let us note that under $\xi_{2}=0$

$$
\left(F_{1} T_{\varphi}^{-1} u\right)\left(\xi_{1}, 0\right)=\tilde{u}\left(\xi_{1}, 0\right) .
$$

For convenience we re-denote the operator $F_{1} T_{\phi}^{-1}$ and introduce the operator

$$
(M \tilde{u})\left(\xi_{1}, \xi_{2}\right)=\frac{\tilde{u}\left(\xi_{1}+a \xi_{2}, \xi_{2}\right)+\tilde{u}\left(\xi_{1}-a \xi_{2}, \xi_{2}\right)}{2}
$$




$$
+v \cdot p \cdot \frac{i}{2 \pi} \int_{-\infty}^{+\infty} \frac{\tilde{u}\left(\eta, \xi_{2}\right) d \eta}{\xi_{1}+a \xi_{2}-\eta}-v \cdot p \cdot \frac{i}{2 \pi} \int_{-\infty}^{+\infty} \frac{\tilde{u}\left(\eta, \xi_{2}\right) d \eta}{\xi_{1}-a \xi_{2}-\eta} \equiv\left(V_{\varphi}^{-1} \tilde{u}\right)(\xi) .
$$

For function of three variables we consider the cone

$$
C_{+}^{a b}=\left\{y \in \mathbb{R}^{3}: y=\left(y_{1}, y_{2}, y_{4}\right), y_{3}>a\left|y_{1}\right|+\left|y_{2}\right|, a, b>0\right\} .
$$

Let us introduce the following one-dimensional singular integral operators $[5,7]$

$$
\left(S_{1} u\right)\left(\eta_{1}, \eta_{2}, \eta_{3}\right)=v \cdot p \frac{i}{2 \pi} \int_{-\infty}^{+\infty} \frac{u\left(\tau, \eta_{2}, \eta_{3}\right) d \tau}{\eta_{1}-\tau}, \quad\left(S_{2} u\right)\left(\eta_{1}, \eta_{2}, \eta_{3}\right)=v \cdot p \frac{i}{2 \pi} \int_{-\infty}^{+\infty} \frac{u\left(\eta_{1}, \tau, \eta_{3}\right) d \tau}{\eta_{2}-\tau} .
$$

and write using calculations from [11]

$$
\begin{gathered}
F_{2} T_{\psi}^{-1} u(\eta)=\left(K_{1} \tilde{u}\right)\left(\eta_{1}-a \eta_{3}, \eta_{2}-b \eta_{3}, \eta_{3}\right)+\left(K_{2} \tilde{u}\right)\left(\eta_{1}-a \eta_{3}, \eta_{2}+b \eta_{3}, \eta_{3}\right) \\
+\left(K_{3} \tilde{u}\right)\left(\eta_{1}+a \eta_{3}, \eta_{2}-b \eta_{3}, \eta_{3}\right)+\left(K_{4} \tilde{u}\right)\left(\eta_{1}+a \eta_{3}, \eta_{2}+b \eta_{3}, \eta_{3}\right)
\end{gathered}
$$

where $K_{1}-K_{4}$ are integral operators of the following type:

$$
\begin{gathered}
\left(K_{1} \tilde{u}\right)\left(\eta_{1}-a \eta_{3}, \eta_{2}-b \eta_{3}, \eta_{3}\right)=\frac{1}{4} \tilde{u}\left(\eta_{1}-a \eta_{3}, \eta_{2}-b \eta_{3}, \eta_{3}\right)-\frac{1}{2}\left(S_{1} \tilde{u}\right)\left(\eta_{1}-a \eta_{3}, \eta_{2}-b \eta_{3}, \eta_{3}\right) \\
-\frac{1}{2}\left(S_{2} \tilde{u}\right)\left(\eta_{1}-a \eta_{3}, \eta_{2}-b \eta_{3}, \eta_{3}\right)+\left(S_{1} S_{2} \tilde{u}\right)\left(\eta_{1}-a \eta_{3}, \eta_{2}-b \eta_{3}, \eta_{3}\right) ; \\
\left(K_{2} \tilde{u}\right)\left(\eta_{1}-a \eta_{3}, \eta_{2}+b \eta_{3}, \eta_{3}\right)=\frac{1}{4} \tilde{u}\left(\eta_{1}-a \eta_{3}, \eta_{2}+b \eta_{3}, \eta_{3}\right)-\frac{1}{2}\left(S_{1} \tilde{u}\right)\left(\eta_{1}-a \eta_{3}, \eta_{2}+b \eta_{3}, \eta_{3}\right) \\
+\frac{1}{2}\left(S_{2} \tilde{u}\right)\left(\eta_{1}-a \eta_{3}, \eta_{2}+b \eta_{3}, \eta_{3}\right)-\left(S_{1} S_{2} \tilde{u}\right)\left(\eta_{1}-a \eta_{3}, \eta_{2}+b \eta_{3}, \eta_{3}\right) ; \\
\left(K_{3} \tilde{u}\right)\left(\eta_{1}+a \eta_{3}, \eta_{2}-b \eta_{3}, \eta_{3}\right)=\frac{1}{4} \tilde{u}\left(\eta_{1}+a \eta_{3}, \eta_{2}-b \eta_{3}, \eta_{3}\right)+\frac{1}{2}\left(S_{1} \tilde{u}\right)\left(\eta_{1}+a \eta_{3}, \eta_{2}-b \eta_{3}, \eta_{3}\right) \\
\quad-\frac{1}{2}\left(S_{2} \tilde{u}\right)\left(\eta_{1}+a \eta_{3}, \eta_{2}-b \eta_{3}, \eta_{3}\right)-\left(S_{1} S_{2} \tilde{u}\right)\left(\eta_{1}+a \eta_{3}, \eta_{2}-b \eta_{3}, \eta_{3}\right) ; \\
\left(K_{4} \tilde{u}\right)\left(\eta_{1}+a \eta_{3}, \eta_{2}+b \eta_{3}, \eta_{3}\right)=\frac{1}{4} \tilde{u}\left(\eta_{1}+a \eta_{3}, \eta_{2}+b \eta_{3}, \eta_{3}\right)+\frac{1}{2}\left(S_{1} \tilde{u}\right)\left(\eta_{1}+a \eta_{3}, \eta_{2}+b \eta_{3}, \eta_{3}\right) \\
+\frac{1}{2}\left(S_{2} \tilde{u}\right)\left(\eta_{1}+a \eta_{3}, \eta_{2}+b \eta_{3}, \eta_{3}\right)+\left(S_{1} S_{2} \tilde{u}\right)\left(\eta_{1}+a \eta_{3}, \eta_{2}+b \eta_{3}, \eta_{3}\right),
\end{gathered}
$$

so we have exact expression for $F_{2} T_{\psi} F_{2}^{-1}=V_{\psi}^{-1}$. Moreover, it was proved in [12] that

$$
\sum_{j=1}^{4}\left(K_{j} u\right)\left(\eta_{1}, \eta_{2}, 0\right)=\tilde{u}\left(\eta_{1}, \eta_{2}, 0\right) \text {. }
$$

\section{BOUNDARY VALUE PROBLEM AND DEGENERATED CONES}

A boundary value problem appears if we need to obtain a unique solution for the equation (1). As we have seen above there is a lot of solutions according to a number of arbitrary functions $c_{k}, k=0,1, \ldots, n$. To extract the unique solution we consider a certain integral condition in latter author's papers [10-13].

The formula includes certain parameters of cones and we interested to know how looks the solution if some parameters tend to infinity. Let us note that if the parameters tend to zero we return to well known cases $[3,9,12,13]$. According to the above sections we consider here the following boundary value problem for a product of the cones $C_{+}^{a} \subset \mathbb{R}^{2}$ and $C_{+}^{a b} \subset \mathbb{R}^{3}$ in 5 -dimensional space $\mathbb{R}^{5}$, namely

$$
\left\{\begin{array}{l}
(A u)(x, y)=0, \quad(x, y) \in \mathbb{R}^{5} \backslash\left(C_{+}^{a} \times C_{+}^{b c}\right) \\
\int_{\mathbb{R}^{2}} u\left(x_{1}, x_{2}, y_{1}, y_{2}, y_{3}\right) d x_{2} d y_{3}=f\left(x_{1}, y_{1}, y_{2}\right) .
\end{array}\right.
$$


Let us denote $\tilde{g}\left(\xi_{1}, \eta_{1}, \eta_{2}\right) \equiv A_{\neq}\left(\xi_{1}, 0, \eta_{1}, \eta_{2}, 0\right) \tilde{f}\left(\xi_{1}, \eta_{1}, \eta_{2}\right)$. We will use the changes of variables

$$
\left\{\begin{array}{l}
\xi_{1}+a \xi_{2}=t_{1} \\
\xi_{1}-a \xi_{2}=t_{2}
\end{array} \quad, \quad\left\{\begin{array}{l}
\eta_{1}+a \eta_{3}=\tau_{1} \\
\eta_{1}-a \eta_{3}=\tau_{3}
\end{array} .\right.\right.
$$

According to expression (3) we introduce the operator

$$
(N \tilde{g})\left(\frac{t_{1}+t_{2}}{2}, \eta^{\prime}\right)=\frac{\tilde{g}\left(t_{1}, \eta^{\prime}\right)+\tilde{g}\left(t_{2}, \eta^{\prime}\right)}{2}+v \cdot p \cdot \frac{i}{2 \pi} \int_{-\infty}^{+\infty} \frac{\tilde{g}\left(\tau, \eta^{\prime}\right) d \tau}{t_{1}-\tau}-v \cdot p \cdot \frac{i}{2 \pi} \int_{-\infty}^{+\infty} \frac{\tilde{g}\left(\tau, \eta^{\prime}\right) d \tau}{t_{2}-\tau} .
$$

Analogously we introduce the operators $(L \tilde{g})\left(\xi^{\prime}, \frac{\tau_{1}+\tau_{3}}{2}, \eta_{2}\right)=K_{1}\left(\xi^{\prime}, \tau_{3}, \eta_{2}\right)+K_{2}\left(\xi^{\prime}, \tau_{3}, \eta_{2}\right)+$ $K_{3}\left(\xi^{\prime}, \tau_{1}, \eta_{2}\right)+K_{4}\left(\xi^{\prime}, \tau_{1}, \eta_{2}\right)$. After accurate evaluations one can obtain

$$
(L \tilde{g})\left(\xi^{\prime}, \frac{\tau_{1}+\tau_{3}}{2}, \eta_{2}\right)=\frac{\tilde{g}\left(\xi^{\prime}, \tau_{1}, \eta_{2}\right)+\tilde{g}\left(\xi^{\prime}, \tau_{3}, \eta_{2}\right)}{2}+\left(S_{1} \tilde{g}\right)\left(\xi^{\prime}, \tau_{1}, \eta_{2}\right)-\left(S_{1} \tilde{g}\right)\left(\xi^{\prime}, \tau_{3}, \eta_{2}\right) .
$$

Theorem 2. Let the symbol $A(\xi)$ admits the wave factorization with respect to the cone $C$ with the index $æ$ such that $æ-s=1+\epsilon,|\epsilon|<1 / 2$ for enough large a. Then for arbitrary $f \in H^{s+1}\left(\mathbb{R}^{3}\right)$ the unique solution of the problem (5) has limit under $a \rightarrow \infty, b=$ const if the function $\tilde{g}$ satisfies the equation for all $\eta_{2} \in \mathbb{R}$

$$
\tilde{g}\left(\frac{t_{1}+t_{2}}{2}, \frac{\tau_{1}+\tau_{3}}{2}, \eta_{2}\right)=(N L \tilde{g})\left(\frac{t_{1}+t_{2}}{2}, \frac{\tau_{1}+\tau_{3}}{2}, \eta_{2}\right) .
$$

Proof. We use the equalities (2) and (4) and assertion of Theorem 1 for $|k|=0$. Then we have only one arbitrary function $\tilde{c}_{0}\left(\xi_{1}, \eta_{1}, \eta_{2}\right), \xi=\left(\xi_{1}, \xi_{2}\right), \eta=\left(\eta_{1}, \eta_{2}, \eta_{3}\right)$. The integral condition from (5) gives the relation $\tilde{u}\left(\xi_{1}, 0, \eta_{1}, \eta_{2}, 0\right)=\tilde{f}\left(\xi_{1}, \eta_{1}, \eta_{2}\right)$, from which we determine immediately

$$
\tilde{c}_{0}\left(\xi_{1}, \eta_{1}, \eta_{2}\right)=A_{\neq}\left(\xi_{1}, 0, \eta_{1}, \eta_{2}, 0\right) \tilde{f}\left(\xi_{1}, \eta_{1}, \eta_{2}\right) .
$$

According to Theorem 1 the unique solution of the problem (5) in $\mathbb{R}^{5} \backslash\left(C_{+}^{a} \times C_{+}^{a b}\right)$ satisfies the equality $A_{\neq}(\xi, \eta) \tilde{u}(\xi, \eta)=\left(V_{\phi \psi}^{-1} \tilde{g}\right)(\xi, \eta)$. Thus, for the left hand side of the latter equality we obtain

$$
A_{\neq}\left(\frac{t_{1}+t_{2}}{2}, \frac{t_{1}-t_{2}}{2 a}, \frac{\tau_{1}+\tau_{3}}{2}, \eta_{2}, \frac{\tau_{1}-\tau_{3}}{2 a}\right) \tilde{u}\left(\frac{t_{1}+t_{2}}{2}, \frac{t_{1}-t_{2}}{2 a}, \frac{\tau_{1}+\tau_{3}}{2}, \eta_{2}, \frac{\tau_{1}-\tau_{3}}{2 a}\right),
$$

so that under $a \rightarrow \infty$ we obtain

$$
A_{\neq}\left(\frac{t_{1}+t_{2}}{2}, 0, \frac{\tau_{1}+\tau_{3}}{2}, \eta_{2}, 0\right) \tilde{u}\left(\frac{t_{1}+t_{2}}{2}, 0, \frac{\tau_{1}+\tau_{3}}{2}, \eta_{2}, 0\right)=\tilde{g}\left(\frac{t_{1}+t_{2}}{2}, \frac{\tau_{1}+\tau_{3}}{2}, \eta_{2}\right) .
$$

It is left to define $\lim _{a \rightarrow \infty} V_{\phi \psi}^{-1} \tilde{g}$.

Using the same changes of variables we can conclude that such limit should be the following

$$
(N L \tilde{g})\left(\frac{t_{1}+t_{2}}{2}, \frac{\tau_{1}+\tau_{3}}{2}, \eta_{2}\right) \text {. }
$$

It completes the proof.

Remark 3. Let us note that the equation (6) is a two-dimensional functional singular integral equation.

\section{CONCLUSION}

Our considerations show that there are a lot of distinct singularities which can be studied by this method. Our approach permits to construct and to study new singular domains in the theory of pseudodifferential equations. 


\section{REFERENCES}

1. S. A. Nazarov and B. A. Plamenevsky, Elliptic Problems in Domains with Piecewise Smooth Boundaries (Walter de Gruyter, Berlin, 1994).

2. B.-W. Schulze, B. Sternin, and V. Shatalov, Differential Equations on Singular Manifolds; Semiclassical Theory and Operator Algebras (Wiley-VCH, Berlin, 1998).

3. G. Eskin, Boundar Value Problems for Elliptic Pseudodifferential Equations (AMS, Providence, RI, 1981).

4. I. Gohberg and N. Krupnik, One-Dimensional Linear Singular Integral Equations (Birkhäuser, Basel, 1992).

5. S. G. Milkhin and S. Prößdorf, Singular Integral Operators (Akademie, Berlin, 1986).

6. V. S. Vladimiriv, Methods of the Theory of Generalized Functions (Taylor and Francis, London, 2002).

7. F. D. Gakhov, Boundary Value Problems (Dover, Mineola, NY, 1981).

8. N. I. Muskhelishvili, Singular Integral Equations (North-Holland, Amsterdam, 1976).

9. V. B. Vasil'ev, Wave Factorization of Elliptic Symbols: Theory and Applications. Introduction to the Theory of Boundary Value Problems in Non-Smooth Domains (Kluwer Academic, Dordrecht, 2000).

10. V. B. Vasilyev, "Pseudo-differential equations and conical potentials: 2-dimensional case," Opusc. Math. 39, 109-124 (2019).

11. V. B. Vasilyev, "Pseudo-differential equations, wave factorization, and related problems," Math. Meth. Appl. Sci. 41, 9252-9263 (2018).

12. V. B. Vasilyev, "On certain 3D limit boundary value problem," Lobachevskii J. Math. 41 (5), 913-921 (2020).

13. V. B. Vasilyev, "Boundary value problems for elliptic pseudodifferential equations in a multidimensional cone," Differ. Equat. 56, 1324-1334 (2020).

14. V. B. Vasilyev, "Towards the theory of boundary value problems on non-smooth manifolds," AIP Conf. Proc. 2325, $020002(2021)$. 